\title{
Taller de Escritura Creativa para el desarrollo de la Competencia Literaria en Brasil
}

\author{
Julia HABA OSCA \\ Universitat de València \\ Dep. de Filologia Anglesa i Alemanya \\ julia.haba@uv.es \\ María ALCANTUD DÍAZ \\ Universitat de València \\ Dep. de Didàctica de la Llengua i la Literatura \\ maria.alcantud@uv.es \\ Joan PEREDO HERNÁNDEZ \\ IES Joaquín Sorolla, Valencia \\ peredo@alumni.uv.es
}

Recibido: 10 de febrero de 2015

Aceptado: 3 de marzo de 2015

\section{RESUMEN}

Este artículo describe una propuesta de innovación docente basada en la corriente educativa de la Educación para el Desarrollo, así como la mejora de Competencia Comunicativa en L2 y de las Competencias Literarias e Interculturales por medio de un taller diseñado para tal fin. El propósito de este artículo es doble: por un lado mostrar las posibilidades que ofrece el Taller de Escritura e Ilustración Creativa para el desarrollo de las Competencias Literaria, Intercultural y Comunicativa en L2. Se muestra cómo el taller cumple con las directrices marcadas por la Educación para el Desarrollo que se describe en el marco teórico. El segundo objetivo es narrar cómo se han organizado, coordinado e implementado el Taller de Escritura e Ilustración Creativa en la Universidade Federal do Amazonas en Manaos (Brasil), basándose en la metodología del aprendizaje basado en tareas, y cómo se ha conseguido (i) promover la creación de puentes para la consolidación de las relaciones bilaterales entre universidades; (ii) motivar la colaboración científica con los centros brasileños que cuentan con un departamento de español, y (iii) emplear y crear herramientas que permitan incluir la Educación para el Desarrollo.

Palabras clave: Educación para el Desarrollo, Aprendizaje basado en tareas, escritura creativa, taller, Competencia Literaria

\section{Creative Writing Workshop for the development of Literary Competence in Brazil}

\section{ABSTRACT}

This article describes a proposal of teaching innovation based on the teaching stream Education for Sustainable Development, as well as the improvement of the Communicative 
Competence in ESL and both Literary and Intercultural Competences by means of a workshop designed with this purpose. Therefore, the purpose of this article is twofold: on one hand, to demonstrate the possibilities that a Creative Writing and Illustration Workshop offers for the development of the Literary, Intercultural and Communicative Competences in ESL. Additionally, we describe in our theoretical framework how this workshop mentioned follows the Development Education guideline. Our second objective is to narrate how we organized, coordinated and held the Creative Writing and Illustration Workshop at the Universidade Federal do Amazonas in Manaus (Brazil), based in the Task-based methodology, and how we have achieved (1) to promote the creation of connections for the consolidation of bilateral agreements between universities; (2) motivate the scientific collaboration with Brazilian institutions that have a Spanish Department, and (3) use and create tools that may include the Development Education methodology.

Key words: Education for Sustainable Development, Task-based learning, creative writing, workshop, Literary Competence

\section{Atelier d’Écriture Créative pour le développement de la Compétence Littéraire à Brésil}

\section{RÉSUMÉ}

Ce document décrit une proposition de formation d'innovation éducative basée sur l'éducation actuelle pour le développement et l'amélioration de la compétence communicative en L2 et des ouvres littéraires et compétence interculturelle à travers un atelier conçu à cet effet. Le but de cet article est double: tout d'abord montrer les possibilités offertes par l'Atelier d'Écriture Créative et Illustration pour le développement de l'alphabétisation et la compétence communicative interculturelle en L2. Il est destiné à montrer comment l'atelier répond aux orientations fixées par le Éducation pour le Développement décrit dans le cadre théorique. Le deuxième objectif est de décrire comment ils sont organisés, coordonnés et mis en œuvre l'Atelier d'Écriture et Illustration Créative à l'Universidade Federal do Amazonas à Manaus (Brésil), basée sur la méthodologie de l'apprentissage basé sur les tâches, et comment elle a été réalisée pour (i) promouvoir la création de ponts pour la consolidation des relations bilatérales entre les universités; (ii) d'encourager la collaboration scientifique avec les centres brésiliens avec un centre de l'espagnol, et (iii) d'employer et de créer des outils pour inclure l'Éducation pour le Développement.

Mots-clés: Éducation pour le Développement, Apprentissage basé sur les tâches, écriture créative, atelier, Compétence Littéraire

SUMARIO: 1. Introducción; 2. Marco Teórico; 2.1. Educación para el Desarrollo; 2.2. Competencia Literaria; 3. Metodología y Justificación; 3.1. Justificación del proyecto; 3.2. Taller de Escritura e Ilustración Creativa en UFAM; 3.2.1. Primera fase del Taller de Escritura e Ilustración Creativa; 3.2.2. Segunda fase del Taller de Escritura e Ilustración Creativa; 3.2.3. Tercera fase del Taller de Escritura e Ilustración Creativa; 4. Resultados y Discusión; 4.1. Obtención de Cuentos de Tradición oral amazónica e Ilustraciones; 4.2. Difusión y sensibilización; 4.3. Cooperación internacional; 5. Conclusiones; 6. Bibliografía. 


\section{INTRODUCCIÓN}

El propósito de este artículo es mostrar las posibilidades que ofrece el Taller de Escritura e Ilustración Creativa para el desarrollo de las Competencia Literaria en L2 que se está llevando a cabo en distintos lugares del mundo y con diferentes colectivos, con el fin de obtener un material didáctico para la mejora de la Competencia Literaria en el aula de inglés como segunda lengua. La justificación de la elección intercultural como pieza básica surge porque la Educación para el Desarrollo da una gran importancia tanto a la profundización en el conocimiento de la diversidad como en las estrategias básicas: resolución de conflictos, inclusión de las minorías en contenidos y procesos educativos, etc. (Banks 1997, Aguado 2003, Banks y Banks 2001, Besalú, 2002, Díaz-Aguado 2002, Mínguez 2012). Se muestra también cómo los talleres cumplen con las directrices marcadas por la Educación para el Desarrollo.

Nuestro segundo objetivo es describir cómo se ha organizado, coordinado e implementado el Taller de Escritura e Ilustración Creativa en la Universidade Federal do Amazonas en Manaus (UFAM), basándonos en la metodología del aprendizaje basado en tareas, y cómo se ha conseguido (i) promover la creación de puentes para la consolidación de las relaciones entre universidades; (ii) motivar la colaboración científica con los centros brasileños, así como (iii) emplear y crear herramientas que permitan incluir la Educación para el Desarrollo.

\section{MARCO TEÓRICO}

\subsection{Educación para el Desarrollo}

La Educación para el Desarrollo es un concepto que engloba la educación en valores: solidaridad, cooperación, no discriminación, respeto y globalización y que cuenta con algo más de cuarenta años de historia. En este sentido se relaciona con la educación para la paz, en los derechos humanos, intercultural, de género, ambiental y para los medios de consumo.

Esta metodología se basa en diversas teorías como son la pedagogía de Freire (2007), las corrientes constructivistas del conocimiento que tienen su origen en la psicología de Piaget (1981), o el modelo de investigación-acción elaborado por Bruner (1997). Siguiendo a Vygotsky (1978), quien consideraba la educación como un proceso de interacción social en el que el adulto guía al aprendiz y le introduce en la cultura, la Educación para el Desarrollo concibe la enseñanza-aprendizaje como un proceso activo y participativo, en el que el docente actúa como facilitador para ayudar en la construcción del conocimiento, partiendo de sus preocupaciones y centros de interés. Como destaca Freire (2007), la educación debe ser un proceso transformador que lleve a los participantes por un recorrido que se inicia en la auto-concienciación y se dirige a la comprensión de los diversos elementos y estructuras que influyen decisivamente en sus vidas. Así, desarrollarán estrategias, habilidades y técnicas para participar de forma responsable en el desarrollo de su comunidad, influir y mejorar la realidad. Según Mesa (2001) en este período se han producido cambios en el concepto de la práctica y en la importancia que se le otorga a este ámbito de actividad. 
Actualmente, la Educación para el Desarrollo se ha convertido en un componente importante de las políticas y estrategias de los diversos actores que integran el sistema de educación y cooperación al desarrollo, al quedar de manifiesto en estos años de experiencia que es imposible cometer acciones que propicien el desarrollo de los estudiantes sin un cambio previo de actitudes y valores que garanticen la consolidación y sostenibilidad de dichas actuaciones. Por lo tanto, definimos la Educación para el Desarrollo como un proceso educativo constante que favorece la comprensión de las interrelaciones económicas, políticas, sociales y culturales entre el Norte y el Sur, promueve valores y actitudes relacionadas con la solidaridad y la justicia social y busca vías de acción para lograr un desarrollo humano y sostenible. Es una educación sociopolítica, cuyo eje es la justicia social, y se basa en un planteamiento constructivista y socio-histórico. Se trata de un proceso que requiere realizarse en el medio-largo plazo, y donde la dimensión cognitiva, actitudes y valores no pueden separarse. Analiza la realidad desde una dimensión global, proporciona criterios analíticos, está abierta a la participación y orientada a la acción (Mesa, 2001).

Según Brislin (1986) y Celorio y López de Munain (2007), la Educación para el Desarrollo no es sólo un método de trabajo o un enfoque educativo, sino un modo de mantener viva la conciencia indignada por las desigualdades existentes, una manera de no deshumanizarnos y defender la dignidad de todas las personas. Es animar a la participación y ayudar a vencer la indiferencia. Por ello, expertos de HEGOA, entre ellos, Celorio y López de Munain (2007) insisten en que la Educación para el Desarrollo tiene la doble tarea de desarticular actitudes y estructuras injustas e insolidarias y facilitar los medios para la construcción de un mundo más equitativo y solidario.

Desde la perspectiva de la didáctica de las lenguas y la literatura, es imprescindible por tanto, aplicar los mecanismos lectores y experimentar la pluralidad de lecturas. Para que el alumno aprenda a comprender, es necesario que la escuela sistematice la ayuda necesaria para la obtención de habilidades propias de la lectura. De ahí que esta vertiente educativa, se sustente sobre dos pilares fundamentales, siendo el primero el aspecto cognitivo o intelectual. Necesitamos conocer, estudiar a fondo y luego analizar los hechos. Los hechos deben estar relacionados con las situaciones locales, que a su vez deben enmarcarse dentro de su contexto global, para garantizar un entendimiento de las distintas situaciones.

Pero el conocimiento por sí mismo no es suficiente, también hay que entender la educación como ámbito de discusión y crítica cultural. Según Marhuenda (1994), se configura básicamente como una crítica de la cultura dominante, así como una creación de espacios para la expresión de otras culturas y la intercomunicación crítica entre ellas. Y por eso, el segundo pilar, de aspecto ético, supone una tarea más exigente para la Educación para el Desarrollo. Consiste en la adquisición de una actitud de solidaridad bien entendida. Se trata de mantener una atención constante hacia la justicia y la dignidad humana. En este sentido, la Educación para el Desarrollo se plantea el reto de ser una invitación al cambio de comportamientos individuales y colectivos, que nos recuerde, por un lado, que nuestras decisiones 
afectan a nuestras vidas, y también a las de los demás; y, por otro, que los ciudadanos tenemos poder y capacidad para influir en el desarrollo solidario de este mundo y hemos de usarlo con responsabilidad.

Esta metodología nos enseña que nuestra sociedad no está limitada a nuestro barrio, a nuestra ciudad, a nuestro país o a nuestra literatura etnocéntrica, sino que abarca toda la humanidad. Se entiende como el proceso de reflexión que debe llevar a los ciudadanos a ser conscientes de su responsabilidad en la construcción de un mundo más justo, en el que todos puedan hacer efectivo su derecho al desarrollo (Boas, 1940). La Educación para el Desarrollo se entiende como un proceso para generar conciencias críticas, hacer a cada persona responsable y activa, a fin de construir una nueva sociedad civil, comprometida con la solidaridad, entendida ésta como corresponsabilidad.

Con la Educación para el Desarrollo se fomenta la participación en propuestas de cambio para lograr un mundo más justo en el que tanto los recursos y los bienes como el poder estén distribuidos de forma equitativa; dota a las personas y a los colectivos de recursos e instrumentos (cognitivos, actitudinales y afectivos) que les permitan incidir en la realidad para transformar sus aspectos más negativos. Finalmente, se logra un desarrollo humano sostenible en el nivel individual comunitario, local e internacional (Argibay, Celorio y Celorio, 2005).

Bloom (1995), Borrego y Parra (1995) insisten que para el cuerpo docente que pretende motivar al alumnado en la Competencia Literaria, la Educación para el Desarrollo es una estrategia imprescindible. Ya que es a través de la Literatura como los estudiantes toman conciencia de que, en primer lugar, somos una manifestación de los valores de solidaridad presentes en nuestra sociedad, que además es nuestro principal capital social y por ello, tenemos la responsabilidad de incrementar este capital y esta es la razón por la que la labor de Educación para el Desarrollo, la sensibilización y la Competencia Literaria debe ser el eje principal de nuestro hacer.

Según Ballester e Ibarra (2009) los educadores interculturales y profesores de lengua extranjera, deberían emplear más la literatura en el área de lenguas extranjeras porque es una herramienta que nos dinamiza interiormente, nos permite ser fermento en la sociedad y nos da fuerza y legitimidad para desarrollar nuestro trabajo como docentes.

\subsection{Competencia Literaria}

Para definir la Competencia Literaria, nos vamos a remitir, en primer lugar, a Hymes (1971) y a su definición de competencia, como la unión del conocimiento y la capacidad de uso. Mediante la introducción de Competencia Comunicativa, rompió la concepción abstracta que apuntaba Chomsky (1988). Esta nueva noción consideraba los componentes socio-comunicativo e histórico cultural como determinantes en la adquisición de las reglas textuales de la Competencia Comunicativa.

En torno el concepto de Competencia Literaria, y con la pretensión de describir las características que la designan, Van Dijk (1980) la entiende como la habilidad y la capacidad de los seres humanos para producir e interpretar textos literarios. Mientras que para Culler (1975) en su obra La Poética Estructuralista, habla de la 
Competencia Literaria como el conjunto de convenciones para leer los textos literarios: centrándose en el aspecto de la recepción de la obra literaria. La Competencia Literaria es un concepto definido de diversas maneras según la autoría, pero los autores se decantan principalmente por "un saber que permite producir y comprender textos" (López Valero, 2000), que hay que fomentar e impulsar y que "de nada servirá el propugnar la adquisición de este tipo de competencia si con posterioridad centramos nuestro trabajo únicamente en aspectos literarios de tipo técnico" (López Valero y Encabo Fernández, 2001).

Ballester e Ibarra (2013a y 2013b) nos recuerdan que desde hace años se realizan, tanto en la educación infantil como primaria, muchas propuestas en relación con las competencias en comunicación literaria y lingüística. De entre ellas podemos citar: los planes de fomento de la lectura y bibliotecas, el aprendizaje cooperativo para la mejora de las destrezas orales y escritas, la producción de una gran diversidad de tipologías de textos en distintas áreas de conocimiento, la utilización masiva y temprana de TIC o también la utilización del portafolio europeo de las diversas culturas, literaturas y lenguas.

Para nosotros la Competencia Literaria está incluida en la Competencia Intercultural, debido a que a partir de ella se trabajan los mismos aspectos que la conforman, y participa de sus características pero, en concreto, en lo que se refiere al plano escrito, encontrándose contenida de alguna manera dentro de ella, lo que la diferencia de la Competencia Comunicativa, en que esta última engloba el plano oral y escrito.

Nuestro objetivo final, es la de contribuir a que los estudiantes desarrollen la Competencia Literaria en lengua extranjera, un aspecto del conocimiento que apenas se trabaja en el aula, contribuyendo por lo tanto a la adquisición de las Competencias Intercultural y Comunicativa, en segunda instancia, al estar contenida en la anterior, para mejorar la capacidad de comunicación intercultural del alumnado. Y que puede ayudar en gran medida a la instrumentalización de la lectura y escritura en lengua extranjera, a través de propuestas creativas y por tanto motivadoras, como pueden ser los Talleres de Escritura e Ilustración Creativa.

\section{METODOLOGÍA Y JUSTIFICACIÓN}

\subsection{Justificación del proyecto}

TALIS (Enseñanza y Adquisición de Competencias Solidarias e Interculturales a través de las Lenguas y la Literatura) es un proyecto de innovación educativa, intercultural y solidario que nació en respuesta a la percepción de una gran falta de asesoría técnica y colaboración de las universidades con diferentes agentes en materia de educación basada en el modelo AICLE/CLIL (aprendizaje integrado de contenido y lenguas). Por esta razón se creó una plataforma que coordina diferentes acciones para fomentar la Educación para el Desarrollo a través de la cultura, utilizando diferentes lenguas extranjeras como herramientas esenciales para trabajar la Competencia Literaria además de la Intercultural y Comunicativa.

Los objetivos de TALIS son: 1) fomentar actividades donde el hecho de aprender una lengua extranjera no resida exclusivamente en el dominio de la Competencia 
Comunicativa sino que ayude también a conocer la cultura a la que esa lengua pertenece; 2) implementar las Competencias Literaria e Intercultural integrando la enseñanza del inglés siguiendo el modelo AICLE (Richards y Rodgers, 2014) y 3) crear una colección de audio-cuentos multilingües en la cual las culturas estén reflejadas.

A partir del interés del Proyecto TALIS en profundizar sobre la Educación para el Desarrollo, surgió la propuesta de crear materiales auténticos, interculturales y multilingües: cuentos creados por alumnado de diferentes nacionalidades y que recogen su cultura a través de talleres de escritura con estudiantes universitarios o de otros colectivos. De aquí nacen los "Cuentos alrededor del Mundo/Stories around the World". Para la elaboración de esta colección de cuentos, se está llevando a cabo la creación, coordinación e implementación de talleres en diferentes lugares del mundo $\mathrm{y}$ con diferentes colectivos.

Con el desarrollo de este proyecto se obtienen cuentos interculturales y multilingües por diferentes vías: en primer lugar, a través de talleres de escritura (en los que no sólo se crean cuentos interculturales, sino que también se recogen relatos de la tradición oral de cada país). En segundo lugar, se obtienen donaciones de cuentos de voluntarios, bien escritos por ellos mismos o recopilados en sus viajes. Estas narrativas se están utilizando, junto con su traducción al español y/o a otras lenguas, para la publicación de libros como material didáctico intercultural. Los fondos recaudados por la venta de los volúmenes en esta colección se están destinando en la actualidad al Fondo Cultural de la ONG VOCES que logra financiar dos proyectos: Jele Kosobé en Bamako (Mali) y Acción Social en España.

\subsection{Taller de Escritura e Ilustración Creativa en UFAM}

La metodología utilizada en esta actividad se ha basado en el desarrollo de la Competencia Comunicativa, más concretamente en el aprendizaje por tareas (TBL). Según Willis (1996) y Richards y Rodgers (2014), el aprendizaje por tareas se refiere al uso de tareas como unidad central de planificación e instrucción en la enseñanza de lenguas. Para la implementación de esta actividad se ha seguido el proceso de tres etapas, según lo indicado por académicos como Prabhu (1987), Skehan (1996) o Lee (2000).

A continuación, detallaremos la aplicación metodológica de estas fases al Taller de Escritura e Ilustración Creativa en la UFAM. La primera fase implica una serie de actividades que tanto el profesorado como el alumnado han de llevar a cabo. De acuerdo con Ellis (2005) "el propósito de la fase pre-tarea es preparar a los estudiantes para realizar la tarea de manera que fomente la adquisición". En esta fase se perfilan los requisitos de la actividad; se hace el cronograma, simulaciones de la tarea como entrenamiento; y, se proporciona el marco teórico. La segunda fase, es la única obligatoria y se centra en la tarea en sí, acometiendo diferentes instrucciones, incluyendo los requisitos necesarios para llevar a cabo la tarea, así como pautas adicionales. La fase final incluye el seguimiento y feed-back sobre el desarrollo de la actividad realizada y sensibilización (Ellis, 2006). 


\subsubsection{Primera fase del Taller de Escritura e Ilustración Creativa}

Para describir la actividad que fue llevada a cabo en Manaos (Brasil), se divide cada fase en dos bloques: profesorado y alumnado, puesto que cada uno de los colectivos ejerce un papel diferente en cada fase. Este proyecto se ha centrado en actividades cuyo fin es el de preservar y difundir la identidad cultural y lingüística local, por este motivo, el objetivo fue elaborar una compilación de cuentos plurilingües (inglés/portugués; portugués/español; inglés/español) que rescaten del imaginario colectivo cuentos de tradición oral amazónica.

\section{Profesorado}

Se llevaron a cabo los preparativos antes del desplazamiento al terreno por la coordinadora del Proyecto TALIS y los profesores que iban a colaborar en este proyecto. Lo fundamental fue la elaboración de materiales específicos para el correcto desarrollo del taller. Las tareas previas incluyeron la recopilación de datos, configuración del equipo de trabajo y diseño del itinerario de viaje.

Una vez llegados los profesores españoles a la Universidade Federal do Amazonas (UFAM), comenzaron las labores de coordinación del desarrollo del Taller de Escritura e Ilustración Creativa: localización de espacios para impartir el curso y del profesorado que iba a realizar el taller. Se preparó el material de aula, y se procedió a la localización del alumnado por medio de una convocatoria para esta actividad y una definición previa de perfil de alumnado. Los requisitos que debían de cumplir los alumnos para poder participar en el taller fueron los siguientes: 1) que quisieran participar como voluntarios, 2) que fueran estudiantes de diseño en la UFAM en activo y 3) que firmaran un compromiso de permanencia en el proyecto durante la duración del mismo. Una vez seleccionado el alumnado participante, se llevó a cabo el registro de inscripciones e información a las personas seleccionadas para desarrollar el curso, así como las labores logísticas para la coordinación del aula y horarios para el desarrollo del taller. La duración del proyecto fue de 3 semanas. Participaron un total de 40 estudiantes (de 60 que asistieron a la presentación inicial del proyecto) y 7 profesores.

\section{Estudiantes}

Se presentó en primer lugar el proyecto TALIS, su origen y la manera en la que está creando un fondo cultural en favor de la ONG VOCES destinado a la financiación de proyectos de cooperación internacional relacionados con el arte y la educación. También se presentaron dos libros publicados: Cuentos alrededor del Mundo (Alcantud-Díaz 2014a y Alcantud-Díaz 2014b), fruto de otros talleres organizados anteriormente en otros países. Se describieron las características de los relatos (originales o adaptados de la tradición oral, relativamente breves, contextualizados en el Amazonas, que traten los ejes temáticos de la Educación para el Desarrollo) y se organizaron grupos, para fomentar el trabajo cooperativo. De esta forma, solicitando al alumnado que observara un modelo de cómo llevar a cabo la tarea, se logró mantener la capacidad de atención y se involucraba a los asistentes en 
la primera fase diseñada para que llevaran a cabo su tarea que consistió en la realización de un cuento y dos ilustraciones.

\subsubsection{Segunda fase del Taller de Escritura e Ilustración Creativa}

\section{Profesorado}

El Taller de Escritura e Ilustración Creativa fue guiado mediante un PowerPoint que el proyecto TALIS tiene preparado en varios idiomas para este fin. En este caso estaba traducido al portugués y contenía toda la información y pautas necesarias. Además, se llevaron a cabo los registros fotográfico y documental. El material recopilado incluye el registro de cuentos elaborados por el alumnado (texto, ilustraciones, datos de los estudiantes), y pequeños videos que muestran la historia de todo el proceso de creación. Asimismo, se creó una cuenta grupal en Facebook que servía como plataforma educativa virtual y vínculo permanente tanto con el alumnado como con el profesorado participante para colgar noticias diversas, vídeos diarios, recoger opiniones, etc.

\section{Estudiantes}

Evidentemente, la tarea se llevó a cabo bajo presión de tiempo, éste es una de las características del TBL (Ellis, 2006). Siguiendo a Lee (2000), se decidió establecer un estricto control de tiempo porque se contaba solamente con un período de 21 días para la realización del taller.

El alumnado siguió el taller aprendiendo y haciendo de manera simultánea, el método learning by doing de Schank (1995). En primer lugar se efectuó una sesión de lluvia de ideas para así obtener material con el que poder crear sus propios cuentos. Después llegó el momento de tomar decisiones: qué tipo de cuento se deseaba crear (amor, fantasía, drama, infantil, comedia, misterio, etc.); el título; y qué valores se iban a transmitir (social, solidario, género, relaciones norte-sur, ambiental, cultural, etc.). Además, se les aconsejó que para fomentar las competencias relacionadas con la investigación, usaran diccionarios o recursos online para resolver sus dudas o incrementar el número de palabras o expresiones de campos semánticos específicos (tradiciones, festivales, gastronomía, etc.).

\subsubsection{Tercera fase del Taller de Escritura e Ilustración Creativa}

Según Ellis (2005) esta fase persigue el cumplimiento de tres objetivos pedagógicos principales: 1) dar una oportunidad para revisar o repetir la puesta en marcha de la tarea, 2) invitar a la reflexión sobre la manera en que la tarea se ha llevado a cabo; y, 3) llamar la atención sobre la forma de la tarea, especialmente en aquellas que son de difícil puesta en marcha.

\section{Profesorado}

La post-tarea del profesorado consistió en la edición y montaje del material recopilado en Brasil. Además, a lo largo de los meses siguientes a la ejecución del taller, se llevó a cabo la labor de difusión del proyecto en España con noticias en 
prensa $^{1}$ y la organización de unas jornadas de sensibilización, la publicación de los cuentos y las ilustraciones obtenidos dentro del marco del proyecto Cuentos alrededor del Mundo.

\section{Estudiantes}

Se intentó obtener con el alumnado el cumplimiento de los tres objetivos pedagógicos marcados por Ellis (2005) mencionados anteriormente. Se revisaron las tareas en grupos, se expusieron los diseños creados con los cuentos, e intercambiaron impresiones y técnicas. También se les invitó a la reflexión sobre la manera en que el taller se había llevado a cabo y sobre los productos finales obtenidos. Actualmente se continúa contactando con los estudiantes voluntarios a través de la página de Facebook creada.

\section{RESULTADOS Y DISCUSIÓN}

\subsection{Obtención de Cuentos de Tradición oral amazónica e Ilustraciones}

En el taller se crearon 10 cuentos y 20 ilustraciones que van a formar parte del próximo volumen de Cuentos alrededor del mundo. En la tabla 1 se puede ver el título de los cuentos y su adecuación temática a la Educación para el Desarrollo, tomando como base la definición que proporciona UNICEF (1993): “...proceso educativo destinado a fomentar actitudes y valores (solidaridad, igualdad, respeto al medioambiente) y los capacita para promover el cambio (institucional, local...)”.

\begin{tabular}{|c|c|c|c|c|c|c|}
\hline $\begin{array}{c}\text { Cuento } \\
\text { Amazónico }\end{array}$ & Sostenibilidad & $\begin{array}{c}\text { Educación } \\
\text { para la paz }\end{array}$ & Género & $\begin{array}{c}\text { Educación } \\
\text { intercultural }\end{array}$ & Pobreza & $\begin{array}{c}\text { Diferencias } \\
\text { norte } \mathrm{y} \text { sur }\end{array}$ \\
\hline $\begin{array}{c}\text { As } \\
\text { Amazonas }\end{array}$ & $\mathrm{x}$ & $\mathrm{x}$ & $\mathrm{x}$ & $\mathrm{x}$ & \\
\hline Boitatá & $\mathrm{x}$ & $\mathrm{x}$ & & $\mathrm{x}$ & $\mathrm{x}$ & \\
\hline Guaraná & $\mathrm{x}$ & $\mathrm{x}$ & & & $\mathrm{x}$ & \\
\hline Iara & $\mathrm{x}$ & $\mathrm{x}$ & $\mathrm{x}$ & & $\mathrm{x}$ & \\
\hline Mapinguari & $\mathrm{x}$ & & & & $\mathrm{x}$ & $\mathrm{x}$ \\
\hline $\begin{array}{c}\text { Matinta } \\
\text { Pereira }\end{array}$ & & $\mathrm{x}$ & $\mathrm{x}$ & $\mathrm{x}$ & $\mathrm{x}$ & $\mathrm{x}$ \\
\hline Pirarucu & $\mathrm{x}$ & $\mathrm{x}$ & & & & \\
\hline Tucumao & $\mathrm{x}$ & $\mathrm{x}$ & & $\mathrm{x}$ & $\mathrm{x}$ & \\
\hline Uirapuru & $\mathrm{x}$ & $\mathrm{x}$ & & $\mathrm{x}$ & & \\
\hline Vitória Régia & $\mathrm{x}$ & $\mathrm{x}$ & $\mathrm{x}$ & $\mathrm{x}$ & & \\
\hline
\end{tabular}

Tabla 1: Clasificación basado en contenidos ED.

Como se aprecia en la tabla, la categoría educativa de la Sostenibilidad, Educación para la Paz y la Pobreza aparecen en más del 70\% de los relatos. Algunos ejemplos son:

\footnotetext{
${ }^{1}$ http://www.elmundo.es/blogs/elmundo/voces/2014/10/07/mucho-mas-que-un-rio.html
} 
“Algunos dicen que para los crímenes imperdonables, la Boitatá era igualmente impiadoso, robando las almas de los criminales y transformándoles en llamas para su cuerpo."

(Boitatá - Educación para la Paz)

"Ellas poseían sus propias reglas y cada una de ellas tenía sus deberes: las mayores tenían pajas para producir cestos y hamacas, y las más jovencitas cuidaban de la agricultura y de cazar."

(As Amazonas - Sostenibilidad)

"Orgullosos de ellos mismos por haber conseguido lo que les habían pedido, se fueron de regreso a su tribu. Pero de camino a casa, el más pequeño de los tres se quejó: - ¡Tengo hambre y no tenemos nada para comer!”

(Tucumao - Pobreza)

Por otra parte, la Educación intercultural la tratan el $60 \%$ de los relatos. En cuanto a las categorías de Género y Diferencias de Norte y Sur, aparecen con menor frecuencia en los cuentos de tradición oral amazónica porque no son categorías que se hayan considerado de especial relevancia con el paso del tiempo según comentaron los participantes en la tercera fase (feed-back) de la tarea. En el caso de la categoría de Género en concreto, la figura de la mujer amazónica siempre ha aparecido como fuerte, independiente y valiente, como se observa en el ejemplo a continuación:

"Ellas preparaban comidas y bebidas apetitosas que emanaban un olor delicioso desde metros de distancia. Las guerreras se embellecían con unas plumas coloridas, unas largas, otras más cortas y voluminosas."

(As Amazonas - Género)

En realidad, la relevancia de estos resultados reside no solo en el contenido de estos relatos, sino en que la metodología de este proyecto hace que formen parte de un fondo intercultural compartido en cada taller que se imparte alrededor del mundo. Por lo tanto, se produce una transmisión cíclica de conocimiento, cultura y valores.

\subsection{Difusión y sensibilización}

En la tercera fase del taller se contemplan las labores de sensibilización y difusión, tanto las que se llevaron a cabo en Brasil, como las que se siguen realizando a propósito de este proyecto. Para sensibilizar tanto a la comunidad universitaria como a la no universitaria de Brasil y España se ha obtenido un registro fotográfico de todo el proceso formativo del cual se puede ver parte en la cuenta de Facebook creada. Se organizó también una jornada de sensibilización en la Universidad de Valencia, donde tuvo lugar una exposición fotográfica del taller, además de las ilustraciones de los cuentos y de los libros editados. 


\subsection{Cooperación internacional}

El beneficio obtenido por la venta de la colección "Cuentos alrededor del Mundo/Stories around the World", forma parte del fondo cultural VOCES como apoyo a la Escuela Kalaban-Coro que dicha entidad tiene en Bamako (Mali). Esta escuela introduce la educación artística como elemento diferencial. La música, el teatro, la danza, contribuyen a mejora la autoestima, la socialización en niños en riesgo social, favoreciendo su recuperación psico-afectiva y dotándolos de herramientas que les permitan enfrentarse a situaciones adversas con creatividad, empatía, iniciativa y seguridad en sí mismos.

Este mismo fondo además apoya el programa de Acción Social en España que favorece la inclusión de los jóvenes en riesgo de exclusión en diversos barrios madrileños. Un plan educativo que combina actividades de refuerzo escolar con actividades socioculturales tales como música, artes plásticas, cine y el circo. A través de las actividades artísticas los participantes pueden crear, innovar y expresarse en libertad; sentirse identificados con diversas comunidades y poder participar en ellas plenamente; tomar conciencia sobre sus capacidades y sobre sus retos; mejorar su autoestima y su socialización.

\section{CONCLUSIONES}

Los talleres de escritura creativa son una propuesta educativa innovadora con gran proyección que ha ido adquiriendo protagonismo durante las últimas décadas (Haba Osca, 2014), situándose, hoy en día como una de las actividades más fructíferas. Este artículo describe una iniciativa de innovación docente basada en la Educación para el Desarrollo, así como la mejora de la Competencia Literaria en L2 y las Competencias Comunicativa e Intercultural por medio de un Taller de Escritura e Ilustración Creativa que tuvo lugar en la Universidade Federal do Amazonas en Manaos (Brasil).

Los resultados de este proyecto, fruto de la colaboración entre profesores y voluntarios de dos países (Brasil y España), vienen a confirmar que los talleres de escritura son una actividad educativa que sirven para promover la consolidación de las relaciones entre universidades y motivar la colaboración científica entre los centros educativos. Por otro lado, permiten emplear y crear herramientas que permiten fomentar la enseñanza y aprendizaje de lenguas y de la cultura a la que dichas lenguas pertenecen, basadas sobre todo en cuentos plurilingües interculturales, sin excluir otras posibilidades, especialmente en el ámbito creativo. Además, el diálogo que se genera entre estudiantes y profesores dentro del curso-taller da lugar a procesos de enseñanza e intercambios lingüísticos que sirven no solamente para establecer colaboraciones en diversos ámbitos sino también que contribuyen a dar a conocer a los jóvenes la magia de la literatura. 


\section{BIBLIOGRAFÍA}

AguAdO, María Teresa (2003): Pedagogía intercultural. Madrid, McGraw-Hill.

AlCANTUD-DÍAZ, María (2014a): Cuentos alrededor del Mundo - Stories around the World, Volumen 1: Proyecto Mali. Madrid, Vaughan Systems.

AlCANTUD-DíAZ, María (2014b): Cuentos alrededor del Mundo - Stories around the World, Volumen 2: Proyecto España. Madrid, Vaughan Systems.

ArgiBAY, Miguel, CELORIO, Gema y Celorio, Juan José (1997): Educación para el desarrollo. El espacio olvidado de la cooperación. Vitoria-Gasteiz, HEGOA.

BALLESTER, Josep e IBARRA, Noelia (2009): "La enseñanza de la literatura y el pluralismo metodológico". OCNOS: Revista de Estudios sobre la Lectura, Cuenca, UCLM, 5, 25-36.

BALLESTER, Josep e IBARRA, Noelia (2013a): "La tentación diabólica de instruirse. Reflexiones a propósito de la educación lectora y literaria". OCNOS: Revista de Estudios sobre la Lectura, Cuenca, UCLM, 10, 7-26.

BALLESTER, Josep e IBARRA, Noelia (2013b): “Interculturalidad". En MARTOS, E. y CAMPOS, M. (ed). Diccionario de nuevas formas de lecturas y escritura. Madrid: Red Internacional de Universidades Lectoras/Santillana, 330-334.

BANKS, Cherry A. y BANKS, James Albert (2001): Multicultural Education: Issues and Perspectives. Boston, Allyn and Bacon.

BANKS, James Albert (1997): Educating Citizens in a Multicultural Society. New York, Teachers College Press.

BESALÚ, Xavier (2002): Diversidad cultural y educación. Madrid, Síntesis.

BESALÚ, Xavier (2007): Educar en sociedades pluriculturales. Barcelona, Wolters Kluwer.

BLOOM, Harold (1995): El canon occidental. Barcelona, Anagrama.

BOAS, Franz (1940): Race, Language and Culture. New York, McMillan.

Borrego ZAPATA, Víctor Manuel y PARRA MEMBriVes, Eva (1995): "El Alumno como receptor literario. Condicionamientos de la lectura e interpretación de un texto literario en clase". En GUERRERO RUÍz, P. y LÓPEZ VALERO, A. (1995) (eds.): Aspectos de Didáctica de la Lengua y la Literatura. Actas del III Congreso Internacional de la Sociedad Española de Didáctica de la Lengua y la Literatura. Murcia, Servicio de Publicaciones Universidad de Murcia, 601-607.

BRISLIN, Richard W. (1986): Cross-Cultural Encounters. Face-to-Face Interaction. Nueva York, Pergamon Press.

BRUNER, Jerome (1997): La educación,puerta de la cultura. Madrid, Aprendizaje Visor.

CElorio, Gema y LóPez de MunAIn, Alicia (2007): Diccionario de educación para el desarrollo. Vitoria-Gasteiz, HEGOA.

CHOMSKY, Noam (1988): El lenguaje y los problemas del conocimiento. Madrid, Visor.

CULLER, Jonathan (1975): La poética estructuralista. El estructuralismo, la lingüistica y el estudio de la literatura. Barcelona, Anagrama.

DíAZ-AGUADO, María José (2002): Educación intercultural y aprendizaje cooperativo. Madrid, Pirámide. 
ELlis, Rod (2005): Planning and Task Performance in a Second Language. Philadelphia, John Benjamin Publishing.

ELLIS, Rod (2006): "The Methodology of Task-Based Teaching". The Asian EFL Journal, 8, 3-13.

FREIRE, Paulo (2007): Pedagogía de la Esperanza. México, Siglo Veintiuno Editores.

HABA OsCA, J. (2014): Taller de Poesía en el área de inglés como lengua extranjera: Hacia el desarrollo de las Competencias Literaria, Intercultural y Comunicativa. Valencia, tesis doctoral.

HYMES, Dell (1971): "Competence and performance in linguistic theory". En HuXley, R. e IngRAM, E. (coords). Language Acquisition: Models and Methods. London, Academic Press, 3-28.

LEE, James (2000): Tasks and Communicating in Language Classrooms. Boston, McGraw-Hill.

LÓPEZ VAlero, Amando (2000): Didáctica de la Lengua y la Literatura. Murcia, ICE-Universidad de Murcia.

LÓPEZ VALERO, Amando y ENCABO FERnÁNDEZ, Eduardo (2001): "El taller de escritura en Educación Secundaria". En Educar en el 2000. Revista de Formación del Profesorado. Lectura y escritura. Murcia, Servicio de Formación del Profesorado Centros de Profesores y Recursos, 1.

MESA, Manuela (2001): "Reflexiones sobre el modelo de las Cinco Generaciones de Educación para el Desarrollo". Education Global Research, 161-167.

MARHUENDA, Fernando (1994): La Educación para el desarrollo en la escuela. Posibilidades e interrogantes. Barcelona, Intermón.

MínGUEZ, Xavier (2012): "La definición de la LIJ en el paradigma de la Didáctica de la Lengua y la Literatura". AILIJ, 10, 87-105.

PiAget, Jean (1981): La teoría de Piaget. Monografias de infancia y aprendizaje. Barcelona, Gedisa.

PRABHU, N.S. (1987): Second Language Pedagogy. Oxford, Oxford University Press.

RICHARDS, J. (1996): “Teachers' maxims in language teaching”. TESOL Quarterly, 30, 281-296.

RICHARDS, Jack C. y RoDGERS, Theodore S. (2014): Approaches and Methods in Language Teaching. Cambridge, Cambridge University Press.

SCHANK, Roger C. (1995): "What We Learn When We Learn by Doing”. Retrieved from the internet 02/12/2014. http://cogprints.org/637/1/LearnbyDoing_Schank.html

SKEHAN, Peter (1996): "A framework for the implementation of task-based instruction". Applied Linguistics, 17, 38-62.

UNICEF (1993): Programa de aprendizaje permanente. Bruselas, UNICEF.

VAN DiJK, Teun (1980): Texto y contexto. Madrid, Cátedra.

Vygotsky, Lev Semiónovich (1978): Pensamiento y lenguaje, Madrid, Paidós.

WILliS, Jane (1996): A Framework for Task-Based Learning. Longman Handbooks for Language Teachers. Harlow, Longman. 